\title{
Hydro-Damage Properties of Red-Bed Mudstone Failures Induced by Nonlinear Seepage and
}

\section{Diffusion Effect}

First Author/Corresponding Author 1\#: Zhenni Ye

Title: Doctor

Affiliation: School of Civil Engineering and Architecture, Hainan University, Haikou, Hainan, 570228, China.

E-mail: zn.ye@hainanu.edu.cn

Co-corresponding Author 2\#: Xiaoli Liu, Associate professor, Tsinghua University.

Title: Professor

Affiliation: State Key Laboratory of Hydroscience and Engineering, Tsinghua University, Beijing, 100084, China.

E-mail: xiaoli.liu@mail.tsinghua.edu.cn

\section{Author 3\#: Enzhi Wang}

Title: Professor

Affiliation: State Key Laboratory of Hydroscience and Engineering, Tsinghua University, Beijing, 100084, China.

E-mail: nzwang@mail.tsinghua.edu.cn

\section{Author 4\#: Huan Sun}

Title: Associate professor

Affiliation: School of Civil Engineering and Architecture, Hainan University, Haikou, Hainan, 570228, China.

E-mail: sunhuan@hainanu.edu.cn

\section{Co-corresponding Author 3\#: Qinxi Dong}

Title: Professor

Affiliation: School of Civil Engineering and Architecture, Hainan University, Haikou, Hainan, 570228, China.

E-mail: dongqinxi@,hainanu.edu.cn 


\section{Hydro-Damage Properties of Red-Bed Mudstone Failures Induced by Nonlinear Seepage and Diffusion Effect}

Abstract: Nonlinear catastrophes caused by geological fluids are a fundamental issue in rock mechanics and the geoengineering hazard field. For the consideration of hydrodynamic force on red-bed mudstone softening damage, $\mathrm{X}$-ray visualization test on the fissure flow in mudstone block failure under hydrodynamic force was performed in this study based on block scale and the physical phenomena of fissure seepage and nonlinear diffusion were further explored. A new method for evaluating the hydro-damage degrees of rocks using an X-ray image analysis was proposed, and the quantitative relation of diffusion coefficients of hydro-damage and seepage was established. The research results revealed that the hydrodynamic force promoted the fluid-filled fissure behavior in mudstone specimen failure. Also, the seepage and diffusion phenomena of fluid in rocks during failures were indicated using X-ray imaging. A dual mechanical behavior was presented in the nonlinear seepage and abnormal diffusion of a red mudstone geological body under hydrodynamic conditions. The damaged degree of mudstone was aggravated by the effect of hydrodynamic force, and the initial seepage-diffusion coefficient with respect to lower hydro-damage was larger than the final seepage-diffusion coefficient with respect to higher hydro-damage of rocks with a decreasing nonlinear trend.

Keywords: hydro-damage, red-bed mudstone, hydrodynamic, X-ray image, seepage-diffusion

\section{Introduction}

Geologic disasters introduced by soft rocks of red-bed areas have been frequently reported in Southwest China [1], especially landslide hazards caused by heavy rainfall [2 2,3$]$, which are prolific .The side slope damage seepage of mudstone geologic bodies intensified by the hydrodynamic force is a fundamental cause of landslide hazards. The lithology of geologic bodies in a red-bed mountainous area is mainly mudstone, shale, and silty mudstone. These lithologic materials are characterized by perviousness, hydrophilicity, softening in water, and easy disintegration during water loss [4], because red-bed soft rock is rich in viscosity soil minerals, such as kaolinite and montmorillonite, which leads to rapid disintegration and softening under hydraulic action. In addition, the redbed soft rock was formed in a hot and high-pressure sedimentary environment and the original joints and fissures in the rock mass were abnormal and sufficient. Under engineering excavation and unloading, fissure development intensifies with a large opening and long extension depth, which often generates the hydraulic channel of soft rock catastrophes. Therefore, heavy rainfall scouring causes the side slope rock mass to create headrace channels. Therefore, drainage must be considered in soft rock reinforcement projects of red beds. Figure 1a presents the failure of a sand and mudstone slope of the Xiashaximiao village in a section from +610 to $+828 \mathrm{~m}$ of the main line K123 of the Mabian highway in Renmu, Sichuan Province. Scouring by heavy rainfall created headrace channels, and the resulting hydrodynamic force intensified the instability of the red soft rock slope induced by the aggravation of hydrodynamic conditions, causing side slope instability of a large area of the highway (as shown in Figure 1b), which directly caused economic loss to projects and gravely threatened the traffic safety of the highway. 

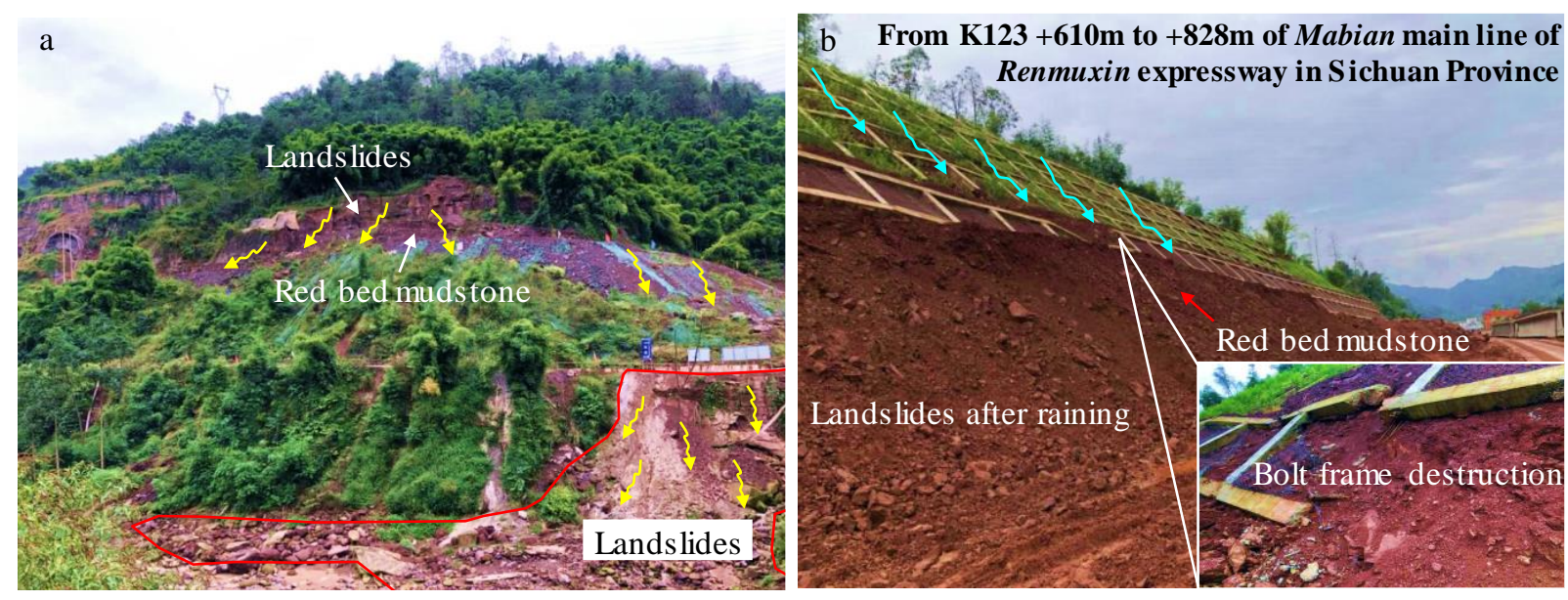

Figure 1 Characteristics of geologic landslide and engineering catastrophes of red-bed soft rocks in Southwest China. (a) Red-bed landslide after rainfall. (b) Side slope support failure of the Renmuxin highway.

The published research on water-rock interactions of red-bed soft rock catastrophes mainly focused on microscopic fissures, disintegration and softening, and damage seepage. Zhou, et al. [5] established the relationship between multi-fractal characteristics and mechanical properties in the consideration of internal fissure structure complexity of soft rock in red beds. The permeability characteristics of red-bed mudstone are closely related to its damage mechanics properties [ㅇ,7]. Various experimental studies on the stress-seepage coupling problems of rocks have been conducted $[\underline{5}, \underline{8-10}]$. The permeability characteristics of rocks under different working conditions have become a typical research topic in rock mechanics and engineering fields, and an important approach has been provided by numerical simulation technology to study such problems. $\underline{\mathrm{Lu}}[10]$ analyzed and predicted the underground water gushing disaster caused by disturbance and damage of a Karst collapse column under the premise of the changing stress and fissure network using the stress-damage-fracture coupling model. The aforementioned research aimed at the permeability characteristics of rocks or rock mass and mainly targeted static or dynamic seepage. In fact, the engineering rock mass introduces dual mechanical behaviors of nonlinear seepage and abnormal diffusion because of the change in external stress [11-13], which is also the main cause for the nonlinear catastrophe of engineering geological fluids. To determine the hydraulic disaster mechanism of red-bed soft rock from the perspective of material science, this study focused on the basic scientific problems of damage and seepage characteristics of red-bed mudstone under hydrodynamic conditions and conducted an X-ray visualization test on the fissure flow during red-bed mudstone fracture [14]. Some study results about mechanics, damage and hydro properties of rocks have been revealed by experiments of acoustic emission signals $[\underline{15}, \underline{16}]$, flow rate [17], fissure flow behaviors [18-20] and so on. In this study, the X-ray image features were analyzed through threshold segmentation and an LOG image algorithm, and the physical phenomena of the nonlinear seepage behavior and diffusion during the fracture of red mudstone specimens were explored. Furthermore, a new approach of rock-water damage evaluation based on an X-ray image technology was proposed and the mathematical relationship between the water damage factor and seepage-diffusion coefficient was established [14]. Finally, a scientific explanation for the water damage disaster of a red engineering geological body is provided from a material perspective, which allows for theoretical guidance for the prevention and control of geological disasters in red-bed soft rock.

\section{Experimental Design on Liquid Flow Observation of Rocks Failure}




\subsection{Principles of Hydro-Damage Definition and Rock Mechanic Tests}

The phenomenon of an engineering geologic hazard in red-bed soft rock induced by rainfall indicates that the intensified damage of red-bed mudstone under hydrodynamic force is the primary cause of side slope instability. According to typical damage mechanics, the damage factor of brittle materials can be defined by an elastic strain approach. The calculation formula is as follows [21]:

$$
D=1-\frac{E^{\prime}}{E_{0}}
$$

Where $E_{0}$ is the original elastic modulus of the material (unit: $\mathrm{MPa}$ ) and $E^{\prime}$ is the elastic modulus of the material in damage status. The purpose of this test was to study the damage characteristics of red-bed mudstone under the cyclic condition of hydrodynamic force. Therefore, the elastic modulus of red-bed mudstone under the action of hydrodynamic force can be defined as $E_{s}^{\prime}$ and the expression of the red-bed mudstone damage factor under the condition of hydrodynamic force can be obtained by

$$
D_{s}=1-\frac{E_{s}^{\prime}}{E_{0}}
$$

Where $E_{S}^{\prime} / E_{0}$ the ratio of the elastic modulus is for damaged and undamaged rocks under hydrodynamic cyclic action, indicating that the parameters $E_{s}^{\prime}$ and $E_{0}$ shall be tested under hydrodynamic force.

An evaluation of mudstone damage caused by hydraulic conduction at the material scale was conducted to reveal the damage characteristics of red-bed mudstone material under hydrodynamic force. The experiment was completed on a medical X-ray flat digital imaging platform, which adopted diversion holes in the mudstone specimens to allow for hydrodynamic force circulation conditions on the premise of water flow and to obtain X-ray images of red-bed mudstone seepage damage under different loads, including physical and mechanical parameters. Moreover, the X-ray image features of fissure flow corresponding to different mechanical parameters were analyzed and compared to establish the correlation between mudstone damage characteristics and seepage image features, and a new approach for red-bed mudstone hydro-damage evaluation was introduced.

Rock materials with different mechanical properties have significant differences in X-ray absorption and attenuation degrees. Figure 2a shows the principle of an X-ray plate-scanning test during rock specimen failures, which was mainly intended for stress, hydraulic conduction flow, and X-ray image data. The X-ray images were analyzed, and the pixels of images in the DICM (Digital Imaging and Communications in Medicine) format were read using the MATLAB software, of which each pixel had an independent coordinate $\left(x_{i}, y_{i}\right.$, and value). $x$ and $y$ are the position coordinates of pixels in the image plane window valued by the X-ray absorbed dose corresponding to the pixel positions (Figure 2b). According to published results of tissues and organs, the rock matrix has a greater X-ray absorption dose threshold than that of iodine, bones, and muscles, and generally, the soft rock has lower X-ray absorption than that of hard rock (Figure 2c). In a special case, the red-bed mudstone has higher X-ray attenuation characteristics because of iron and other metal minerals in the sedimentary environment, whereas rock fissures, soft rock strata, and fissure water have lower X-ray absorption dose, especially fissure water. Therefore, it is difficult to observe water filling fissures in rocks using X-ray scanning equipment. As a result, special image enhancement measures are required to improve the X-ray imaging effect of fissure flow to extract and segment different image features using a threshold approach. 


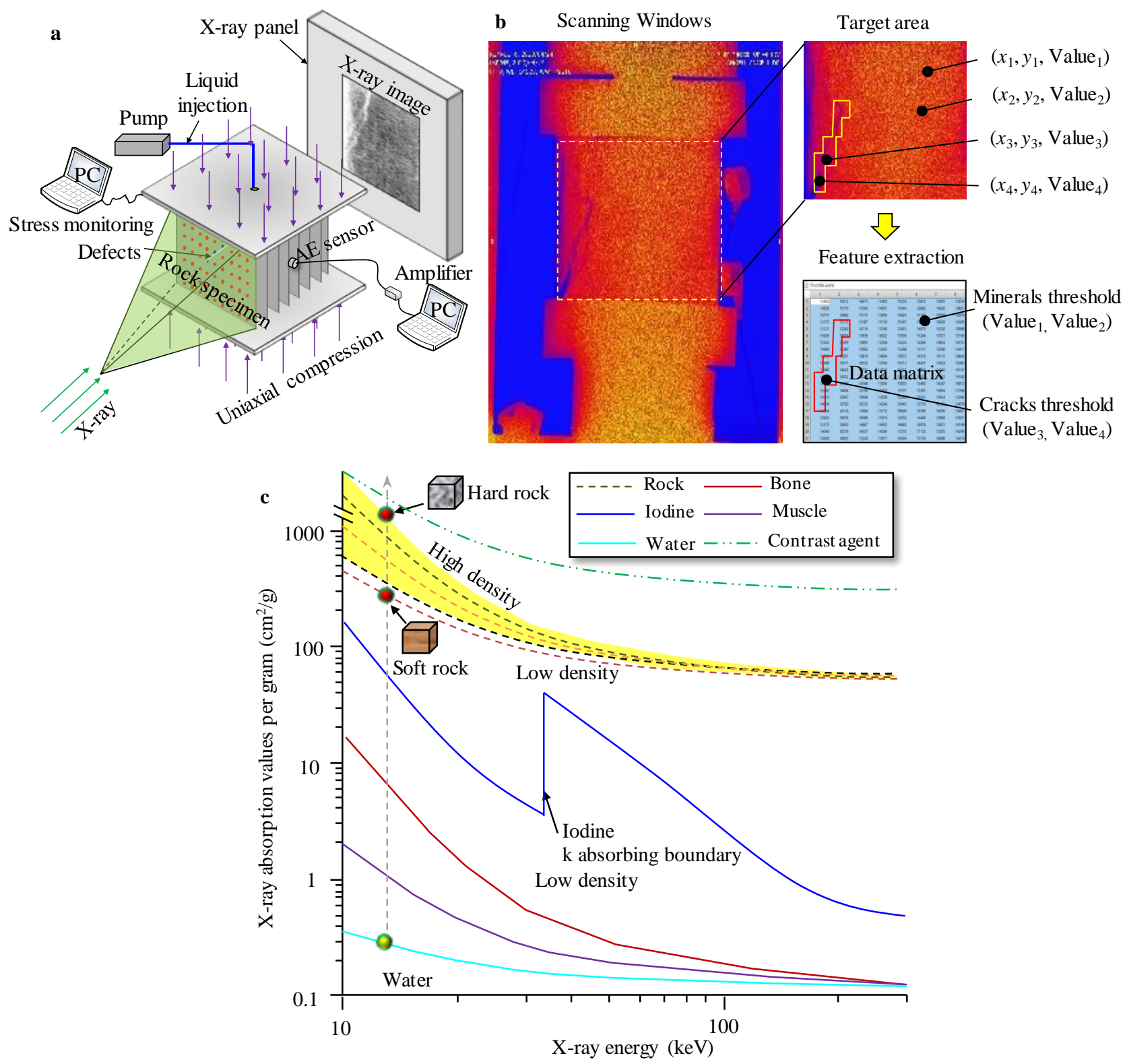

Figure $2 \mathrm{X}$-ray visualization test and image of fissure flow in red-bed mudstone failure under hydrodynamic force. (a) The principle of an X-ray plate-scanning test during rock specimen failures. (b) the X-ray absorbed dose corresponding to the pixel positions. (c) Relations of X-ray absorption dose and X-ray energy for different materials.

\subsection{Enhanced X-ray Image Digital Radiology Techniques and Sample Preparation for Hydro-Rock Testing}

For the X-ray images of fissure flow in red-bed mudstone failure under hydrodynamic conditions, a multiloading rock mechanics testing device matching the X-ray digital radiology imaging platform was designed to simultaneously obtain X-ray images of rock mechanics parameters and fissure flow under pipeline diversion, analyze the influence of multistage loads on the fractured seepage of red mudstone, explore the diffusion effect of red mudstone nonlinear seepage under hydrodynamic conditions, and discuss a new evaluation approach for the degree of mudstone water damage on the basis of the X-ray digital radiology image analysis.

The designed and constructed physical platform is shown in Figure 3. The entire test platform integrated the functions of stress, flow monitoring, and X-ray imaging. The rock specimens were loaded with a hydraulic jack thrust to determine a multistage dynamic load path, and a spoke sensor with a range of 30 tons was selected to collect the stress load. With the help of the data acquisition system, and computer terminal to obtain the stress data 
during mudstone specimen failure. The parameters of the self-priming pump for the contrast fluid diversion of the test are listed in Table 1, and the parameters of the DN4 micro flowmeter for contrast fluid diversion speed monitoring are listed in Table 2. The model of the digital radiology imaging equipment was created as a DSM- 80 flat-panel X-ray machine, which introduced a new type of high-frequency $(100 \mathrm{KHz})$ variable X-ray generator adopting an inverter rectifier module and high-speed rotating anode X-ray tube assembly. To uniformly analyze the X-ray image features of a mudstone specimen failure, the shooting voltage was uniformly set as tube voltage, 80 $\mathrm{kV}$; tube current, $320 \mathrm{~mA}$; and loading time, $400 \mathrm{~ms}$. With the cooperative operation of the test system platform and monitoring, the multivariate mechanical parameters and fissure flow X-ray image data of the rock failure process could be obtained. According to the actual effect of rock failure X-ray imaging, fissure flow had a low degree of absorption attenuation against X-rays; hence, it was difficult to image the feature with a relatively obvious contrast with the fissured medium. Enhanced X-ray image digital radiology (EXIDR) techniques were used for medical cardiovascular imaging with iodine agents with a high attenuation degree to X-rays to improve the imaging quality. To improve and enhance the image quality of the fissure flow in the X-ray scanning system, the contrast medium was used to replace the pure water medium in the experiment. The contrast medium was mainly an aqueous solution, and its hydrodynamic properties were very close to a pure water medium. Meanwhile, there was a difference in the X-ray attenuation between the contrast medium and RBM matrix components; therefore, Xray images with obvious image feature comparisons could be obtained. The comparative images of the meglumine diatrizoate developer and fissure flow before and after X-ray imaging are shown in Figure 4.

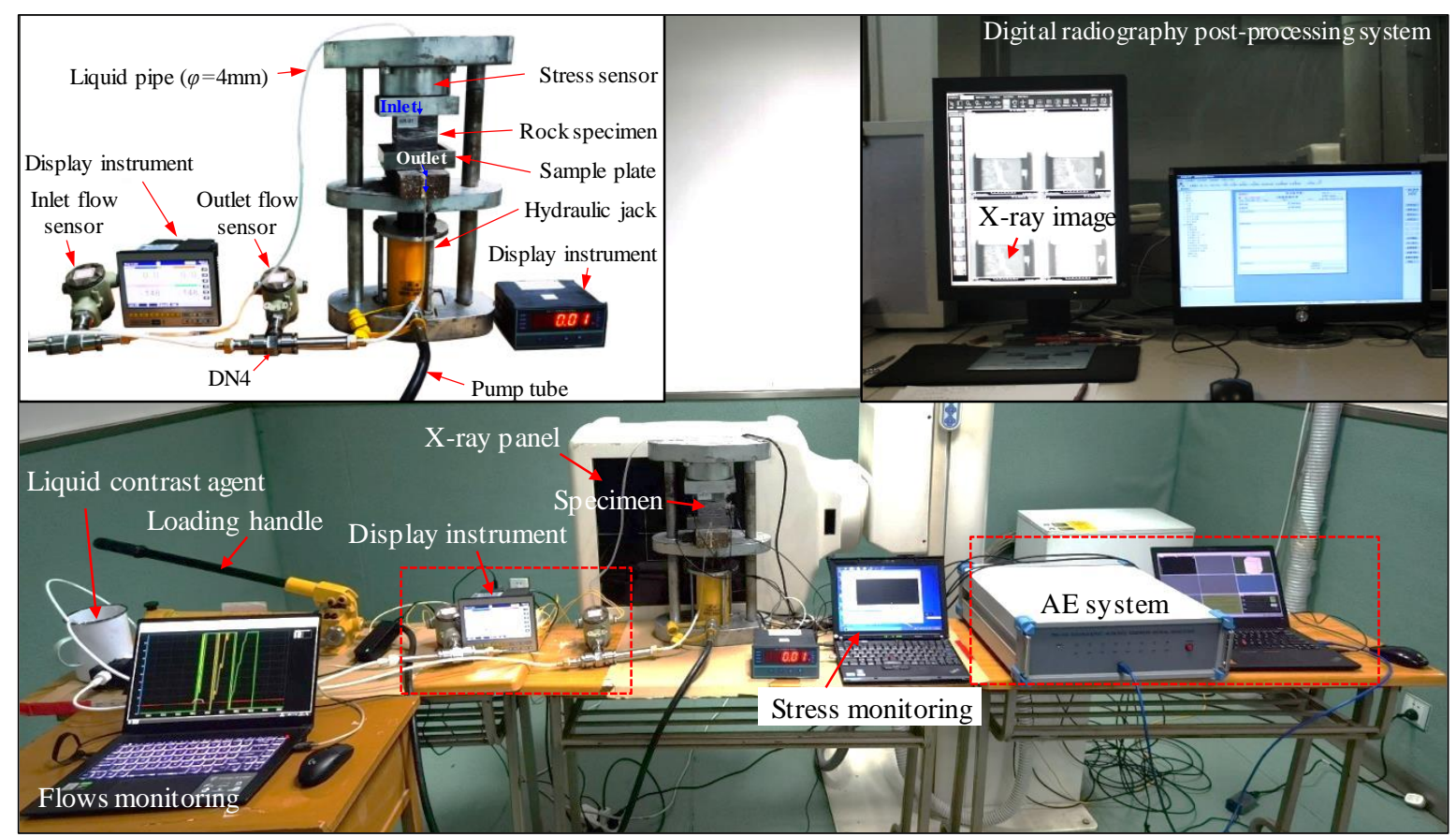

Figure $3 \mathrm{X}$-ray visualization test platform for fissure flow in red-bed mudstone. 


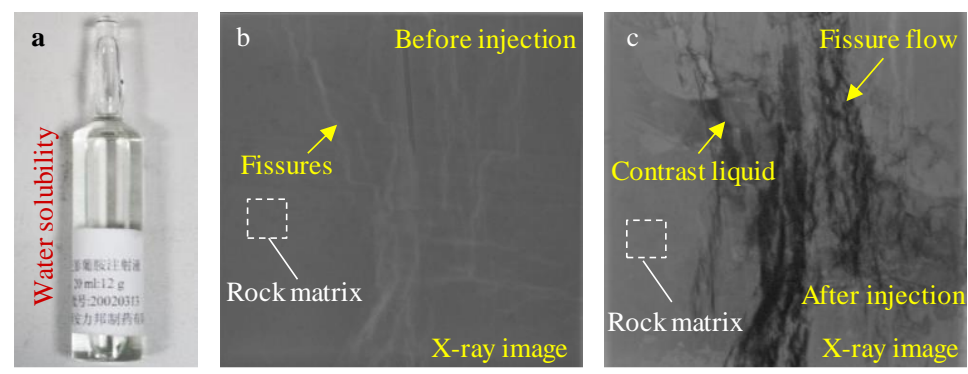

Figure 4 Comparison of meglumine diatrizoate developer and fissure flow before and after X-ray imaging. (a) Water solubility. (b) Xray image feature of rock specimen before contrast liquid injection. (c) Fissure flow visualization in X-ray image of rock specimen after contrast liquid contrast injection.

A test sample was taken from section K2+200 of Minhe Chuanda highway in Haidong, Qinghai Province, China. The sampled mudstone was cut into standard cubic specimens of $100 \times 100 \times 100 \mathrm{~mm}$. Furthermore, a small carborundum drill was also used to make $4.0-\mathrm{mm}$ diameter holes in the center of the top surface of specimens. The sealing joint was set at the diversion interface of a specimen pipeline to avoid leakage during hydraulic transmission. In this experiment, three mudstone specimen cubes were successfully tested (as shown in Figure 5), which were identified as RBM-01, RBM-02, and RBM-03, respectively.

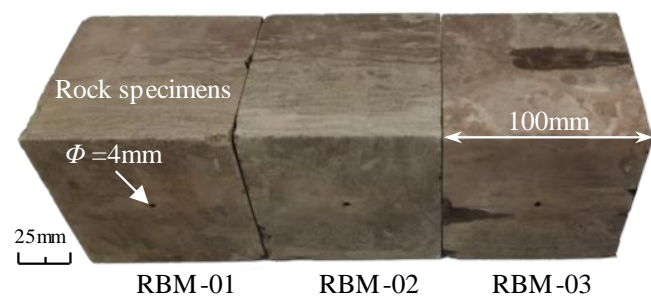

Figure 5 Machining and experimental preparation of the cubic mudstone specimens.

Table 1 Parameters of the Self-Priming Pump

\begin{tabular}{ccccc}
\hline Model & Rated Voltage & Initial Flow & Motor Speed & Maximum Lift \\
\hline LS-0412 & $1.2 \mathrm{~A}$ & $2.0 \mathrm{~L} / \mathrm{min}$ & $2200 \mathrm{r} / \mathrm{min}$ & $30 \mathrm{~m}$ \\
\hline
\end{tabular}

Table 2 Parameters of the DN4 Micro Flowmeter

\begin{tabular}{cccc}
\hline Gauge Diameter $(\mathbf{m m})$ & Normal Flow Range $\left(\mathbf{m}^{\mathbf{3}} / \mathbf{h}\right)$ & Expand Flow Range $\left(\mathbf{m}^{\mathbf{3}} / \mathbf{h}\right)$ & $\begin{array}{c}\text { Typical Withstand Pressure } \\
(\mathbf{M P a})\end{array}$ \\
\hline DN4 & $0.04-0.25$ & $0.04-0.25$ & 6.30 \\
\hline
\end{tabular}

\section{$3 \quad$ Hydro-Damage Evolution with Red-Bed Mudstone Failures}

In this experiment, the X-ray image of fissure flow during mudstone specimen failure was obtained by EXIDR technology, the X-ray image of fissure flow was analyzed to distinguish different components in the mudstone material, and a pixel window with a fixed size of $824 \times 1087$ was used for the X-ray imaging (Figure 6). The accumulated X-ray absorbed dose representing all pixels in the window was denoted as I ( $\mathrm{n}^{\text {th }}$ loading), and the total $\mathrm{X}$-ray absorbed dose corresponding to the fissure flow and rock matrix was obtained by a threshold segmentation approach, which was denoted as $\mathrm{I}_{\mathrm{c}}$ and I, respectively. Sun, et al. [22] confirmed that X-ray absorbed dose was related to rock integrity, elastic modulus parameters, and similar mechanical properties; therefore, there was a positive functional relationship between the elastic modulus $E_{s}$ and the X-ray absorbed dose of mudstone material under the action of hydro-damage $E_{s}=f\left(I_{n}\right)$, where $I_{n}=I_{s}+I_{m}, I_{\mathrm{s}}$ is the X-ray absorbed dose corresponding to the characteristic area of seepage image damaged by mudstone, and $I_{\mathrm{m}}$ accounts for more than $90 \%$ of $\mathrm{I}_{\mathrm{n}}$, which plays a role in controlling rock strength, i.e., $I_{m} \gg I_{s}$. Therefore, Formula 3 can be deduced as follows: 


$$
D_{s}=1-\frac{I_{m}}{I_{0}}
$$

where $I_{\mathrm{m}}$ represents the total X-ray absorption dose (unit: $\mu \mathrm{Gy}$ ) corresponding to the mudstone matrix image area and $I$ represents the total X-ray absorption dose (unit: $\mu \mathrm{Gy}$ ) of the rock matrix in a nondestructive state. In this test, the total absorption dose of all pixel points in the X-ray image obtained under unloaded conditions was recorded as $I_{0}$ and $\mathrm{D}$ was noted as the mudstone damage factor under hydrodynamic force conditions. The calculation results are listed in Table 3.

Figure 6 shows the analytical results of the mudstone matrix color and X-ray absorbed dose before and after the destruction of the RBM-01 specimens. The matrix color of mudstone specimens in a water-saturated state is dark brown. According to the X-ray absorption dose analysis results of the RBM-01 specimens in the saturated hydro-damage state shown in Figure 6, the internal liquid filling fissure of mudstone in the hydro-damage state corresponds to a lower $I_{n}$, i.e., the total X-ray absorption dose $\left(I_{15}\right)$ in the 15th loading complete failure state of RBM-01 specimens is less than the total X-ray absorption dose $\left(I_{0}\right)$ in the nondestructive state. The statistical results of the multilevel stress peak and damage factor calculation during red-bed mudstone damage in this test are listed in Table 4. The resulting X-ray images were segmented according to the threshold to obtain a mudstone matrix and damage seepage characteristics (as shown in Figure 7).
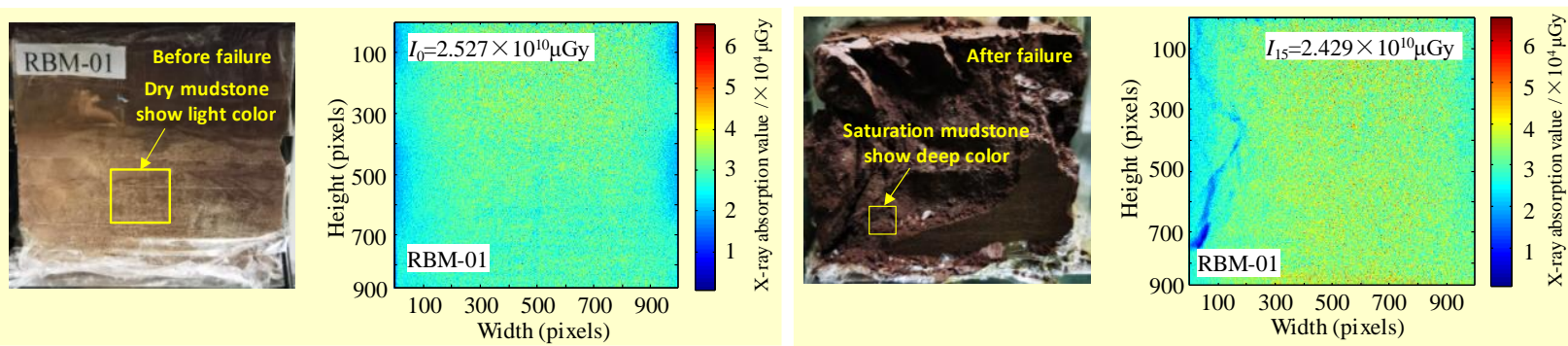

Figure 6 Image interpretations on red-bed mudstone color characteristics and X-ray absorbed dose in nondestructive and hydrodamage states. (a) Color features and image analysis of mudstone surface in nondestructive state. (b) Color features and image analysis of mudstone indication in the hydro-damage state. 

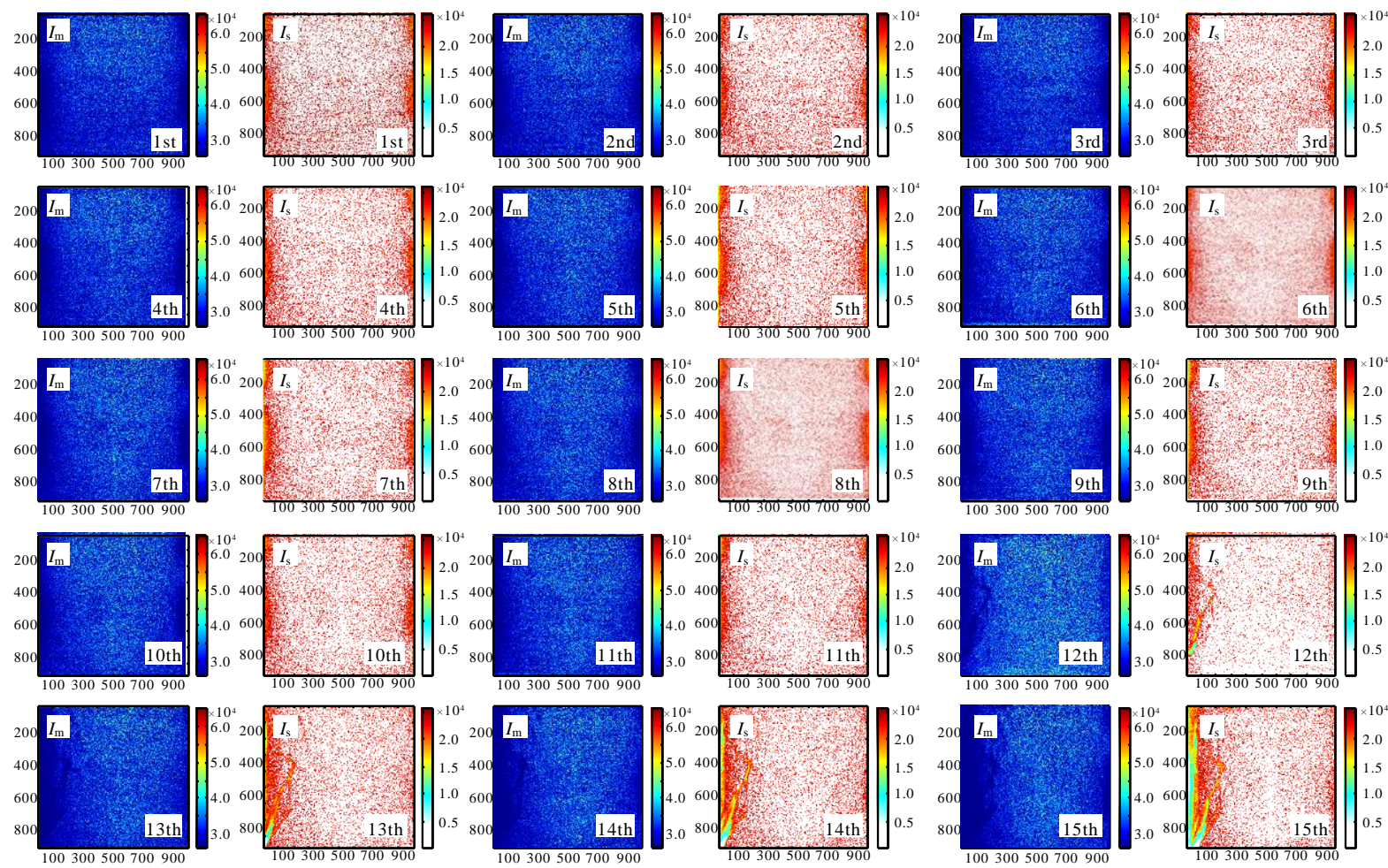

(a) RBM-01 specimens
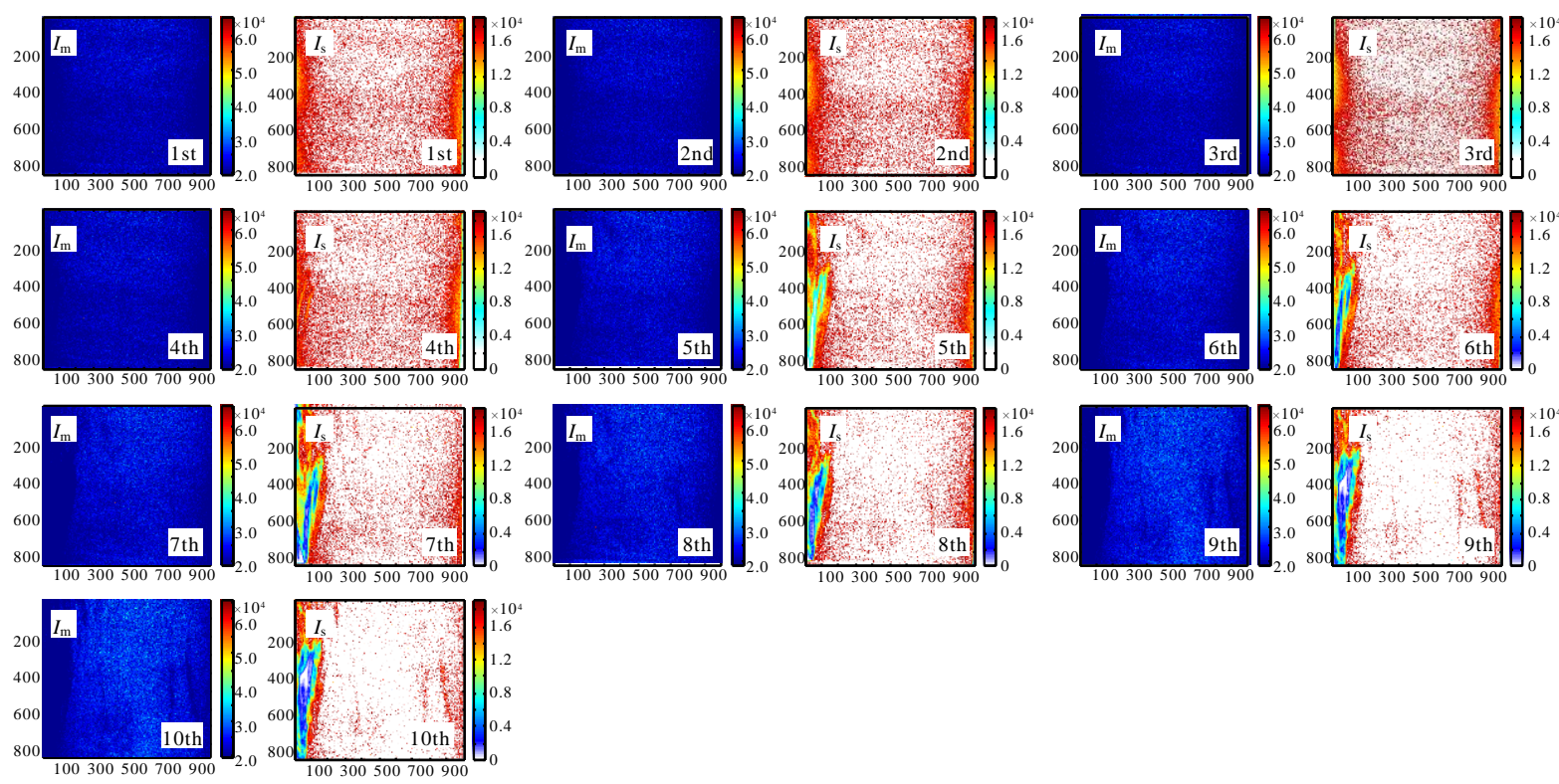

(b) RBM-02 specimens 

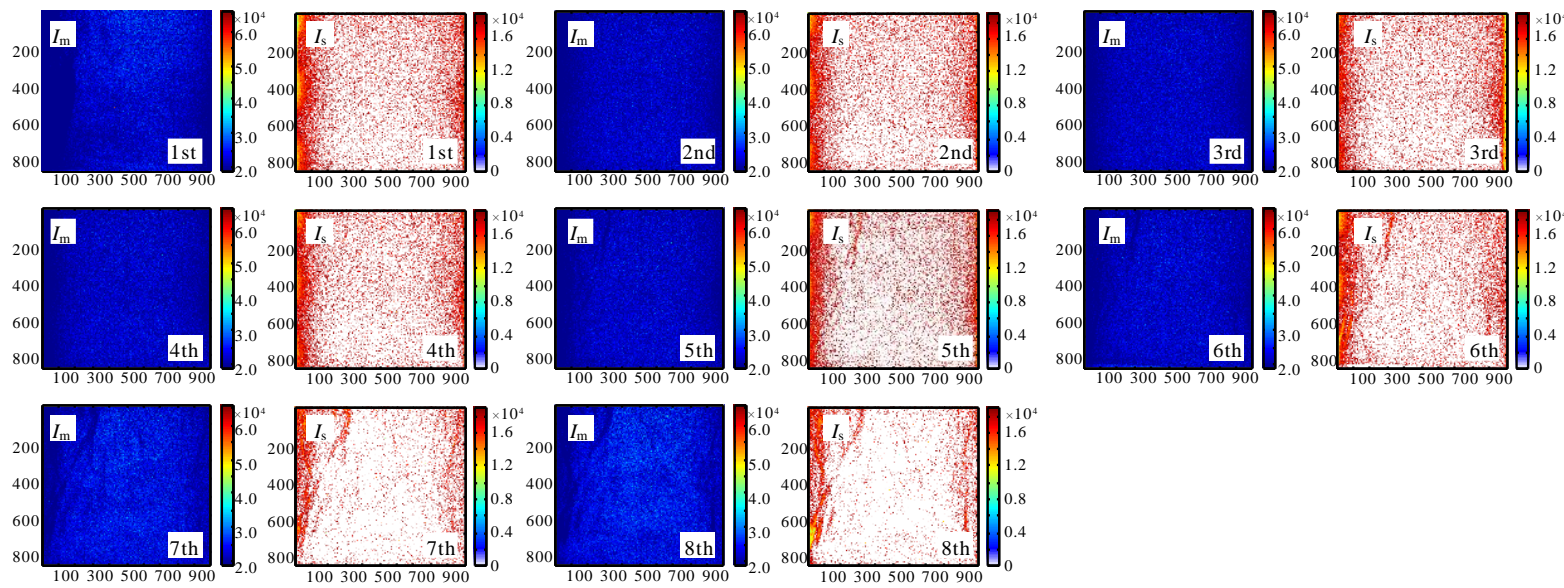

(c) RBM-03 specimens

Figure 7 Image segmentation of red-bed mudstone matrix and damaged seepage features and interpretation on X-ray absorbed dose data.

Table 3 Statistical Results of the Multilevel Stress Peak and Hydro-Damage Coefficient During Red-Bed Mudstone Specimen Failure

\begin{tabular}{|c|c|c|c|c|c|c|c|c|c|c|c|c|c|c|c|}
\hline \multirow{3}{*}{ Number } & \multicolumn{15}{|c|}{ Peak Loading of Multilevel Stress (Mpa) and Hydro-Damage Coefficients } \\
\hline & $\sigma_{1}$ & $\sigma_{2}$ & $\sigma_{3}$ & $\sigma_{4}$ & $\sigma_{5}$ & $\sigma_{6}$ & $\sigma_{7}$ & $\sigma_{8}$ & $\sigma_{9}$ & $\sigma_{10}$ & $\sigma_{11}$ & $\sigma_{12}$ & $\sigma_{13}$ & $\sigma_{14}$ & $\sigma_{15}$ \\
\hline & $D_{\text {s1 }}$ & $D_{\mathrm{s} 2}$ & $D_{\mathrm{s} 3}$ & $D_{\text {s4 }}$ & $D_{\mathrm{s} 5}$ & $D_{\mathrm{s} 6}$ & $D_{\mathrm{s} 7}$ & $D_{\mathrm{s} 8}$ & $D_{\mathrm{s} 9}$ & $D_{\text {s10 }}$ & $D_{\text {s11 }}$ & $D_{\text {s12 }}$ & $D_{\text {s13 }}$ & $D_{\text {s14 }}$ & $D_{\text {s15 }}$ \\
\hline \multirow{2}{*}{ RBM-01 } & 3.82 & 5.56 & 7.66 & 10.12 & 12.76 & 15.58 & 18.20 & 20.74 & 22.32 & 24.16 & 26.44 & 28.52 & 30.96 & 33.30 & 36.76 \\
\hline & 0.354 & 0.364 & 0.372 & 0.391 & 0.408 & 0.405 & 0.413 & 0.413 & 0.518 & 0.622 & 0.625 & 0.629 & 0.731 & 0.843 & 0.907 \\
\hline \multirow{2}{*}{ RBM-02 } & 3.32 & 4.60 & 7.14 & 9.90 & 11.72 & 10.48 & 3.02 & 3.34 & 3.34 & 3.20 & 2.58 & N/A & N/A & N/A & N/A \\
\hline & 0.724 & 0.701 & 0.707 & 0.713 & 0.725 & 0.753 & 0.772 & 0.789 & 0.820 & 0.870 & 0.923 & N/A & N/A & N/A & N/A \\
\hline \multirow{2}{*}{ RBM-03 } & 3.72 & 5.68 & 8.16 & 9.02 & 6.16 & 2.24 & 0.92 & N/A & N/A & N/A & N/A & N/A & N/A & N/A & N/A \\
\hline & 0.444 & 0.448 & 0.714 & 0.811 & 0.942 & 0.948 & 0.972 & N/A & N/A & N/A & N/A & N/A & N/A & N/A & N/A \\
\hline
\end{tabular}

For consideration of the internal mineral composition of each mudstone specimens, the damage seepage image area characteristic threshold of RBM-01 specimens was confirmed as less than $26000 \mu \mathrm{Gy}$ according to the threshold of the digital radiology image scanning system corresponding to the X-ray absorption dose of the mineral composition, and the RBM-02 and RBM-03 components were similar. The characteristic area threshold of damage seepage image $\left(I_{\mathrm{s}}\right)$ was set to less than $19000 \mu \mathrm{Gy}$. Furthermore, MATLAB software was used to statistically analyze corresponding $I_{\mathrm{s}}$ and $I_{\mathrm{m}}$ values of red-bed mudstone specimens under different loads (as shown in Figure 7), and the change law of hydro-damage coefficient $\left(D_{\mathrm{s}}\right)$ in the red-bed mudstone failure under hydrodynamic force was calculated. 

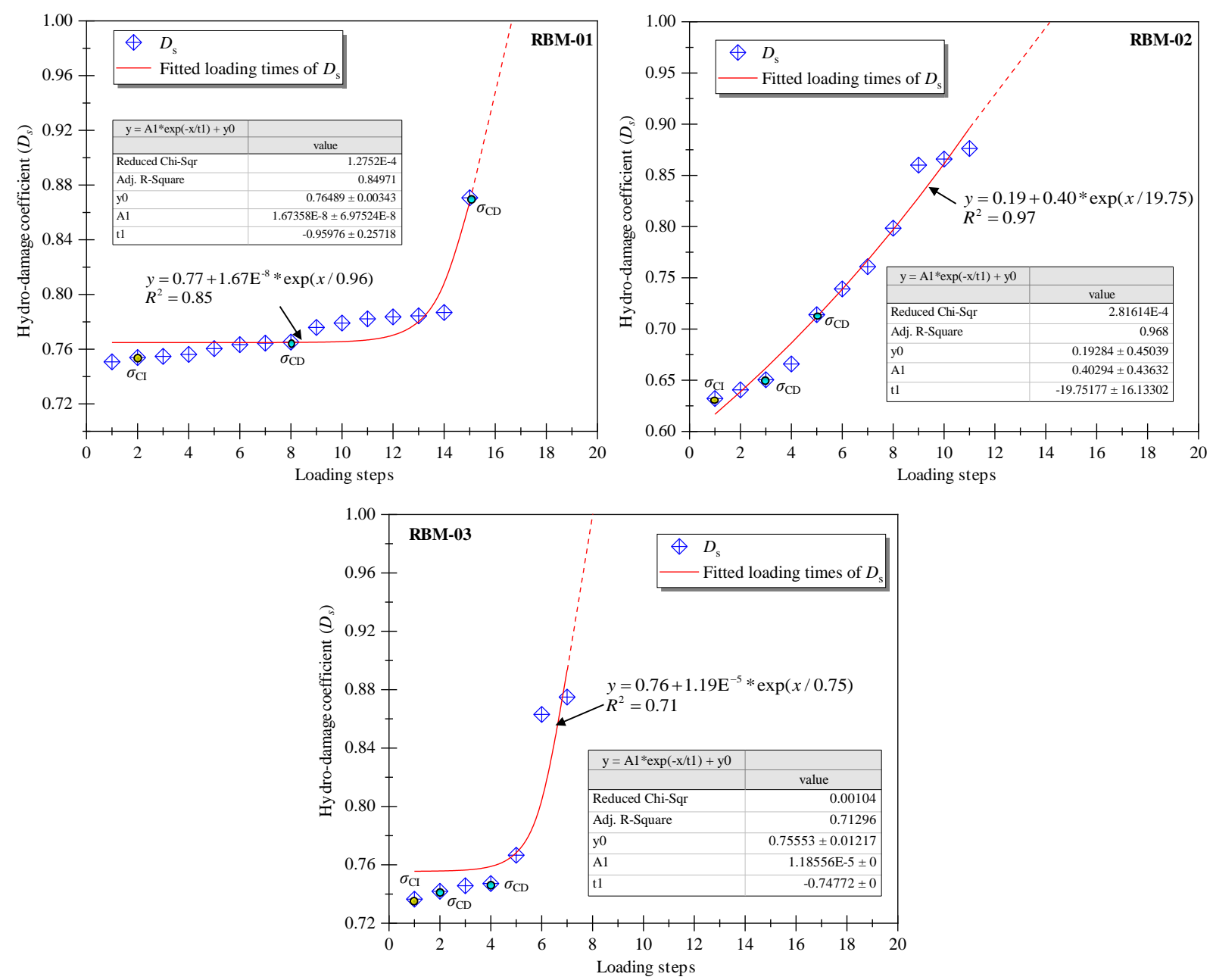

Figure 8 Change law of the hydro-damage coefficient during mudstone specimen failure.

As shown in Figure 8, the change law of the hydro-damage coefficient in the red-bed mudstone specimen failure was evident. The change in the mudstone damage factor under hydrodynamic force has an intensified evolution process. The change in the damage factor of the mudstone specimens exhibits a stable development trend at the initial stage of loading. At this time, the strength damage of the mudstone material intensified, whereas the degree of damage of the hydrodynamic force was not obvious. The hydro-damage coefficient of the mudstone material increased exponentially with the increase in multiload time. The mathematical expression is as follows:

$$
D_{s}=\mathrm{b}+\mathrm{a} \times \mathrm{e}^{\alpha \cdot \mathrm{n}}
$$

Where $\mathrm{a}$ and $\mathrm{b}$ are both constants, $\mathrm{n}$ is the loading time, and $\alpha$ is the coefficient. According to the data fitting results shown in Figure 8, the fitting results $\alpha$ of the RBM-01 and RBM-03 specimens were 1.04 and 1.33, respectively, and the fitting result $\alpha$ of the RBM-02 specimen test was 0.05 . Therefore, when $\alpha>1$, the water damage factor of the mudstone specimen increased nonlinearly with the change in the loading times, whereas when $\alpha<1$, the water damage factor increased moderately. These indicate that the damage effect of the hydrodynamic force on mudstone was a nonlinear process, and the water damage degree of mudstone was mainly affected by the nonlinear seepage behavior and its hydraulics.

\section{Seepage and Diffusion Coefficient Evolution with Rock Failures}

Seepage is the basic mechanical behavior of fluid conduction in a porous medium, and diffusion is a common physical phenomenon of solute transport in a porous medium [23]. The behavior of seepage in the rock dynamic 
failure is often accompanied by a more complicated hydraulic diffusion phenomenon. In this experiment, the image features of mudstone damage seepage at a material scale were obtained by X-ray digital radiology scanning. The behavior of mudstone seepage under hydrodynamic force was accompanied by significant hydraulic diffusion (as shown in Figure 9). Therefore, the X-ray absorption dose threshold approach was applied on the basis of the analysis results from the image of mudstone damage seepage obtained by experiments to segment and calculate the diffusion area of seepage, count the number and actual area of corresponding pixel points in different characteristic regions, and calculate the diffusion coefficient $\left(C_{d}\right)$ of mudstone seepage according to Formula $(5)$.

$$
C_{d}=\frac{S_{\text {total }}}{N_{\text {pixles }}} \cdot \frac{N_{c}}{t}
$$

$C_{\mathrm{D}}$ is the rock seepage-diffusion coefficient (unit: $\mathrm{mm} / \mathrm{s}$ ), which implies the area of seepage and diffusion of fluid in the rock within a unit interval, $S_{\text {total }}$ is the actual total area (unit: $\mathrm{mm}^{2}$ ) corresponding to the segmented X-ray feature images, $N_{\text {pixels }}$ is the total number of pixel points of the segmented X-ray feature images, $N_{c}$ is the total number of pixel points of the seepage-diffusion feature image area, and $t$ is the hydrodynamic force cycle cumulative time (unit: s) corresponding to the X-ray image shooting. The area and pixel points of the feature area shown in Figure 9 were identified and extracted, and the seepage-diffusion coefficient was calculated according to Formula (5). The calculation results are listed in Table 4.
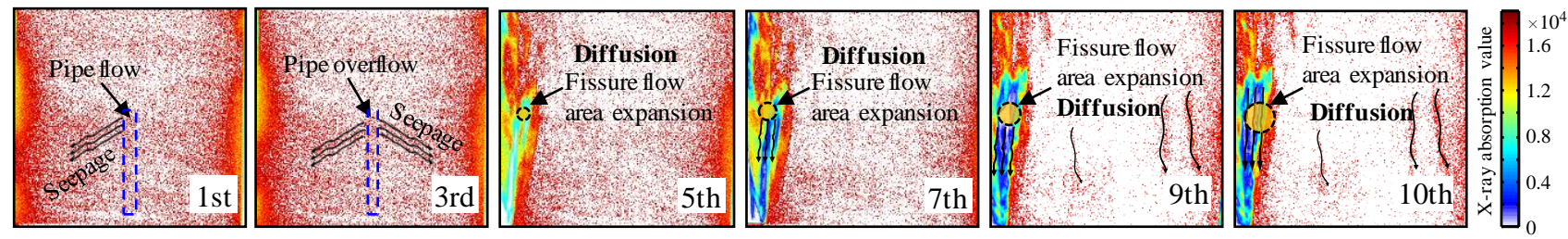

Figure 9 Fissure seepage and diffusion phenomenon in red-bed mudstone failure.

Table 4 Calculation Results of the Seepage-Diffusion Coefficient in Mudstone Specimen Failure

\begin{tabular}{|c|c|c|c|c|c|c|}
\hline \multirow{2}{*}{$\begin{array}{l}\text { Loading } \\
\text { steps }\end{array}$} & \multicolumn{2}{|c|}{ RBM-01 } & \multicolumn{2}{|c|}{ RBM-02 } & \multicolumn{2}{|c|}{ RNM-03 } \\
\hline & $\begin{array}{l}\text { Thresholds } \\
\text { ( } \mu \mathrm{Gy})\end{array}$ & $\begin{array}{l}\text { Seepage-diffusion } \\
\text { coefficient }\left(\mathrm{mm}^{2} / \mathrm{s}\right)\end{array}$ & $\begin{array}{l}\text { Thresholds } \\
\text { ( } \mu \mathrm{Gy})\end{array}$ & $\begin{array}{l}\text { Seepage-diffusion } \\
\text { coefficient }\left(\mathrm{mm}^{2} / \mathrm{s}\right)\end{array}$ & $\begin{array}{c}\text { Thresholds } \\
\text { ( } \mu \mathrm{Gy})\end{array}$ & $\begin{array}{l}\text { Seepage-diffusion } \\
\text { coefficient }\left(\mathrm{mm}^{2} / \mathrm{s}\right)\end{array}$ \\
\hline $1 \mathrm{st}$ & 26000 & 187.69 & 19000 & 274.28 & 19000 & 409.95 \\
\hline $2 \mathrm{nd}$ & 26000 & 16.45 & 19000 & 188.97 & 19000 & 228.74 \\
\hline $3 \mathrm{rd}$ & 26000 & 4.02 & 19000 & 80.51 & 19000 & 92.63 \\
\hline 4 th & 26000 & 2.84 & 19000 & 60.03 & 19000 & 65.33 \\
\hline 5 th & 26000 & 2.01 & 19000 & 48.00 & 19000 & 50.73 \\
\hline 6th & 26000 & 2.04 & 19000 & 36.33 & 19000 & 39.95 \\
\hline 7th & 26000 & 2.01 & 19000 & 28.33 & 19000 & 32.40 \\
\hline 8 th & 26000 & 1.96 & 19000 & 25.43 & 19000 & 29.03 \\
\hline 9th & 26000 & 1.99 & 19000 & 20.82 & N/A & N/A \\
\hline 10th & 26000 & 1.99 & 19000 & 18.48 & N/A & N/A \\
\hline 11 th & 26000 & 1.99 & 19000 & 16.70 & N/A & N/A \\
\hline 12 th & 26000 & 1.77 & N/A & N/A & N/A & N/A \\
\hline 13th & 26000 & 1.77 & N/A & N/A & N/A & N/A \\
\hline 14th & 26000 & 1.81 & N/A & N/A & N/A & N/A \\
\hline 15 th & 26000 & 2.07 & N/A & N/A & N/A & N/A \\
\hline
\end{tabular}



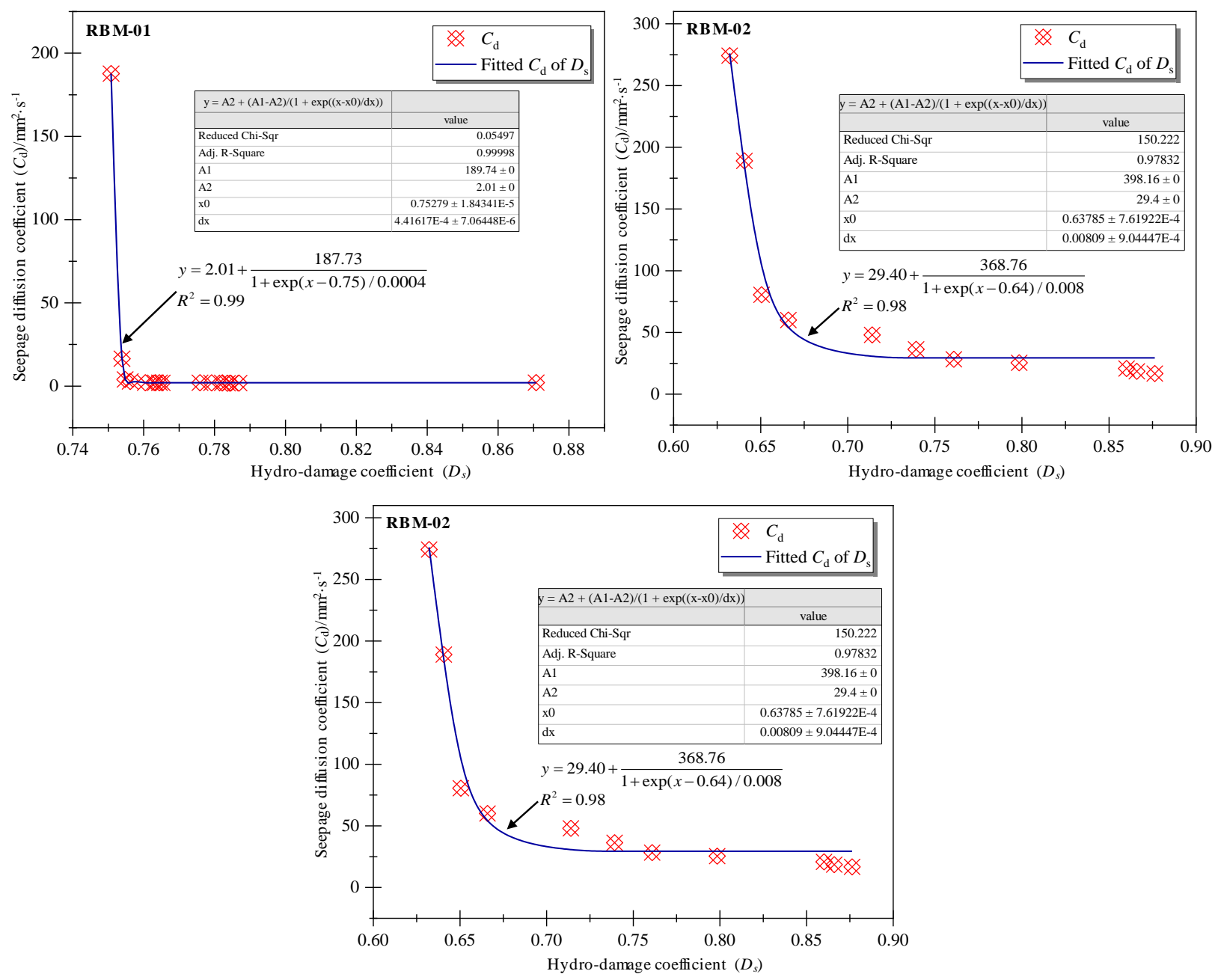

Figure 6 Fitting relation between the hydro-damage factor and seepage-diffusion coefficient.

The relationship between the mudstone hydro-damage factor and seepage-diffusion coefficient were obtained by Boltzmann function fitting, as shown in Figure 10. In the hydro-damage aggravation of the mudstone specimen, the seepage-diffusion coefficient of the mudstone presented a nonlinear decreasing trend, which indicates that the initial seepage velocity and nonlinear diffusion coefficient of the mudstone specimen were relatively high in the initial fissure state. However, the pipeline overflowed, the hydrodynamic action intensified the fluid-filled fissure, and the saturation degree of the mudstone specimen increased gradually against the gradual decrease in the saturated seepage velocity and diffusion flux as the expansion of the internal fracture of the mudstone specimen and the gradual rupture of pipeline. The functional relation between the hydro-damage factor and seepage-diffusion coefficients of the mudstone specimens is as follows:

$$
C_{\mathrm{d}}=a_{0}+\frac{a_{1}}{1+\exp \left[\left(D_{S}-\mathrm{b}\right) / \mathrm{c}\right]}
$$

where $a_{0}, a_{1}, \mathrm{~b}$, and c are all constants. The fitting relation between $D_{\mathrm{s}}$ and $C_{\mathrm{d}}$ indicates that the red-bed mudstone material has greater initial seepage velocity and an abnormal diffusion coefficient, which is a reasonable explanation for the red-bed mudstone geologic body seepage catastrophes from a material scale. Controlling or reducing the seepage-diffusion coefficient is an effective means for red-bed disaster control.

\section{Conclusion}

(1) During the failure of red-bed mudstone specimens, hydrodynamic force intensifies the fluid-filled fissure and the interaction between the fissure flow and solid matrix introduces the dispersion and attenuation of an elastic 
wave. Furthermore, fluid-filled fissure undergoes a stable development process with the crack growth.

(2) The X-ray image analysis of the red-bed mudstone specimen failure indicates that the spatial morphology distribution of the pipeline fluid transition to fissure flow showed the physical phenomena of nonlinear seepage and abnormal diffusion. Therefore, the red-bed mudstone pipeline fluid fissure induces a dual mechanical behavior of nonlinear seepage and diffusion with significant seepage-diffusion effects under hydrodynamic force.

(3) The statistical analysis of the relationship between the mudstone hydro-damage factor and seepagediffusion coefficient indicates that the seepage-diffusion coefficient of the mudstone shows a nonlinear decreasing trend under hydrodynamic force with the increase in water damage aggravation of mudstone specimens; the analysis also indicates that the initial seepage-diffusion coefficient was large under the initial rupture state of the mudstone specimens. The saturation degree of the mudstone specimen increases gradually against the gradual decrease in the saturated seepage velocity and diffusion flux. These results support a reasonable explanation for the seepage catastrophes of red-bed mudstone masses from the material scale.

\section{Acknowledgments}

This study was supported by the National Natural Science Foundation of China (Approval No. 52109120, ), the Hainan Provincial Natural Science Foundation of China (Approval Nos. 521QN203 and 421RC487), the National Key Research and Development Program (Approval Nos. 2018YFC1504801 and 2018YFC1504902), the Open Research Fund Program of the State Key Laboratory of Hydroscience and Engineering (Approval Nos. sklhse-2021-C-02 and sklhse-2021-C-07), the Research Fund for Innovation Platform of Hainan Academician (Approval No. YSPTZX202106) and the Cultivation Fund Program for Youth Teachers in Hainan University (Approval No. HDQN202112).

\section{Reference}

1. Zhang, M.; Yin, Y.P.; Huang, B.L. Mechanisms of rainfall-induced landslides in gently inclined red beds in the eastern Sichuan Basin, SW China. Landslides 2015, 12, 973-983, doi:https://doi.org/10.1007/s10346-015-0611-4.

2. Zhang, S.; Xu, Q.; Hu, Z.M. Effects of rainwater softening on red mudstone of deep-seated landslide, Southwest China. Engineering Geology 2016, 204, 1-13, doi:https://doi.org/10.1016/j.enggeo.2016.01.013.

3. Yu, Y.; Shen, M.F.; Sun, H.Y.; Shan, Y.Q. Robust design of siphon drainage method for stabilizing rainfall-induced landslides. Engineering Geology 2019, 249, 186-197, doi:https://doi.org/10.1016/j.enggeo.2019.01.001.

4. Chen, J.; Dai, F.C.; Xu, L.; Chen, S.; Wang, P.F.; Long, W.; Shen, N.Q. Properties and microstructure of a natural slip zone in loose deposits of red beds, southwestern China. Engineering Geology 2014, 183, 53-64, doi:https://doi.org/10.1016/j.enggeo.2014.10.004.

5. Zhou, C.Y.; Yu, L.; You, F.F.; Liu, Z.; Liang, Y.H.; Zhang, L.H. Coupled seepage and stress model and experiment verification for creep behavior of soft rock. International Journal of Geomechanics 2020, 20, doi:https://doi.org/10.1061/(asce)gm.1943-5622.0001774.

6. Ma, J.J.; Wang, J. A stress-induced permeability evolution model for fissured porous media. Rock Mechanics and Rock Engineering 2016, 49, 477-485, doi:https://doi.org/10.1007/s00603-015-0760-8.

7. Zhu, Y.J.; Liu, X.L.; Wang, E.Z. Influence of impoundment gravity and pore pressure on reactivation of faults. Geomechanics and Geophysics for Geo-Energy and Geo-Resources 2020, 6, doi:https://doi.org/10.1007/s40948$\underline{020-00191-9 .}$.

8. Yang, S.Q.; Tian, W.L.; Jing, H.W.; Huang, Y.H.; Yang, X.X.; Meng, B. Deformation and damage failure behavior of mudstone specimens under single-stage and multi-stage triaxial compression. Rock Mechanics and Rock 
Engineering 2019, 52, 673-689, doi:https://doi.org/10.1007/s00603-018-1622-y.

9. Wen, T.; Tang, H.M.; Huang, L.; Wang, Y.K.; Ma, J.W. Energy evolution: A new perspective on the failure mechanism of purplish-red mudstones from the Three Gorges Reservoir area, China. Engineering Geology 2020, 264, doi:https://doi.org/10.1016/j.enggeo.2019.105350.

10. Lu, Y.L.W., B. Z.; He, M. Q.; Wang, L. G.; Ma, D.; Huang, Z. Prediction of fracture evolution and groundwater inrush from karst collapse pillars in coal seam floors: A micromechanics-based stress-seepage-damage coupled modeling approach. Geofluids 2020, 2020, doi:https://doi.org/10.1155/2020/8830304.

11. Zhu, C.; Xu, X.D.; Wang, X.T.; Xiong, F.; Tao, Z.G.; Lin, Y.; Chen, J. Experimental investigation on nonlinear flow anisotropy behavior in fracture media. Geofluids 2019, doi:https://doi.org/10.1155/2019/5874849.

12. Zhang, Y.Q.; Khorshidian, H.; Mohammadi, M.; Sanati-Nezhad, A.; Hejazi, S.H. Functionalized multiscale visual models to unravel flow and transport physics in porous structures. Water Research 2020, 175, doi:https://doi.org/10.1016/j.watres.2020.115676.

13. Sun, H.; Liu, X.; Ye, Z.; Wang, E. Experimental investigation of the nonlinear evolution from pipe flow to fissure flow during carbonate rock failures. Bulletin of Engineering Geology and the Environment 2021, 80, 4459-4470, doi:https://doi.org/10.1007/s10064-021-02210-9.

14. Sun, H.; Liu, X.; Ye, Z.; Wang, E. A new proposed method for observing fluid in rock fractures using enhanced xray images from digital radiography. Geomechanics and Geophysics for Geo-Energy and Geo-Resources 2021, 8, 10, doi:https://doi.org/10.1007/s40948-021-00310-0.

15. Sun, H.; Liu, X.L.; Zhu, J.B. Correlational fractal characterisation of stress and acoustic emission during coal and rock failure under multilevel dynamic loading. International Journal of Rock Mechanics and Mining Sciences 2019, 117, 1-10, doi:https://doi.org/10.1016/j.ijrmms.2019.03.002.

16. Zhang, Y.L.; Zhao, G.F.; Li, Q. Acoustic emission uncovers thermal damage evolution of rock. International Journal of Rock Mechanics and Mining Sciences 2020, 132, doi:https://doi.org/10.1016/j.ijrmms.2020.104388.

17. Zimmerman, R.W.; Al-Yaarubi, A.; Pain, C.C.; Grattoni, C.A. Non-linear regimes of fluid flow in rock fractures. International Journal of Rock Mechanics and Mining Sciences 2004, 41, 384-384, doi:https://doi.org/10.1016/j.ijrmms.2003.12.045.

18. Rong, G.; Cheng, L.; He, R.H.; Quan, J.S.; Tan, J. Investigation of critical non-linear flow behavior for fractures with different degrees of fractal roughness. Computers and Geotechnics 2021, 133, doi:https://doi.org/10.1016/j.compgeo.2021.104065.

19. Xiong, F.; Wei, W.; Xu, C.; Jiang, Q. Experimental and numerical investigation on nonlinear flow behaviour through three dimensional fracture intersections and fracture networks. Computers and Geotechnics 2020, 121, 103446, doi:https://doi.org/10.1016/j.compgeo.2020.103446.

20. Xiong, F.; Jiang, Q.; Xu, C. Fast Equivalent Micro-Scale Pipe Network Representation of Rock Fractures Obtained by Computed Tomography for Fluid Flow Simulations. Rock Mechanics and Rock Engineering 2021, 54, 937-953, doi:https://doi.org/10.1007/s00603-020-02284-Z.

21. Wang, L.Q.; Yin, Y.P.; Huang, B.L.; Dai, Z.W. Damage evolution and stability analysis of the Jianchuandong Dangerous Rock Mass in the Three Gorges Reservoir Area. Engineering Geology 2020, 265, doi:https://doi.org/10.1016/j.enggeo.2019.105439.

22. Sun, H.; Du, W.; Liu, C. Uniaxial Compressive Strength Determination of Rocks Using X-ray Computed Tomography and Convolutional Neural Networks. Rock Mechanics and Rock Engineering 2021, 54, 4225-4237, doi:https://doi.org/10.1007/s00603-021-02503-1. 
23. Raghavan, R. Fractional derivatives: Application to transient flow. Journal of Petroleum Science and Engineering 2011, 80, 7-13, doi:https://doi.org/10.1016/j.petrol.2011.10.003. 